\title{
Spatial attention can bias search in visual short-term memory
}

\author{
Anna C. Nobre*, Ivan C. Griffin and Anling Rao \\ Brain and Cognition Laboratory, Department of Experimental Psychology, University of Oxford, Oxford, UK \\ Edited by: Robert T. Knight, University of California Berkeley, USA \\ Reviewed by: Silvia Bunge, University of California Berkeley, USA \\ George R. Mangun, University of California Davis, USA
}

\begin{abstract}
Whereas top-down attentional control is known to bias perceptual functions at many levels of stimulus analysis, its possible influence over memory-related functions remains uncharted. Our experiment combined behavioral measures and event-related potentials (ERPs) to test the ability of spatial orienting to bias functions associated with visual short-term memory (VSTM), and to shed light on the neural mechanisms involved. In particular, we investigated whether orienting attention to a spatial location within an array maintained in VSTM could facilitate the search for a specific remembered item. Participants viewed arrays of one, two or four differently colored items, followed by an informative spatial (100\% valid) or uninformative neutral retro-cue (1500-2500 ms after the array), and later by a probe stimulus (500-1000 ms after the retro-cue). The task was to decide whether the probe stimulus had been present in the array. Behavioral results showed that spatial retro-cues improved both accuracy and response times for making decisions about the presence of the probe item in VSTM, and significantly attenuated performance decrements caused by increasing VSTM load. We also identified a novel ERP component $\left(\mathrm{N}_{\mathrm{RS}}\right)$ specifically associated with searching for an item within VSTM. Paralleling the behavioral results, the amplitude and duration of the $\mathrm{N} 3_{\mathrm{RS}}$ systematically increased with VSTM load in neutral retro-cue trials. When spatial retro-cues were provided, this "retro-search" component was absent. Our findings clearly show that the influence of top-down attentional biases extends to mnemonic functions, and, specifically, that searching for items within VSTM can be under flexible voluntary control.
\end{abstract}

Keywords: attention, search, memory, retrieval, ERPs, N3 ${ }_{\mathrm{RS}}, \mathrm{N} 2 \mathrm{PC}, \mathrm{CDA}$

\section{INTRODUCTION}

Top-down control from attentional orienting based on our changing expectations and task goals is recognized to have pervasive effects upon perceptual functions at many levels of stimulus analysis (Kastner and Pinsk, 2004; Luck and Hillyard, 2000; Nobre, 2004). Intensive investigation across diverse methodologies has made significant headway in revealing the neural systems and cellular mechanisms of attentional modulation of perceptual representations (e.g., Luck et al., 2000; Mesulam, 1999; Moore, 2006; Reynolds and Chelazzi, 2004; Rushworth and Taylor, 2006; Serences and Yantis, 2006; Womelsdorf and Fries, 2006).

Extrapolating, it would be reasonable to conjecture that the effects of attentional orienting extend to the domain of memory. Volitional control over long-term memory retrieval is implied by our ability to retrieve facts or episodes that are relevant to our current interest or task goal. Likewise, it is intuitive to propose that attentional orienting can bias our access to the contents of short-term memory ${ }^{1}$, enabling us to focus upon particularly relevant objects or features from those currently maintained on-line. Surprisingly, however, this form of flexibility is not an established feature of most memory models, and very little research has considered the existence or the mechanisms of top-down attentional control over memory representations.

*Correspondence: Anna Christina Nobre, Brain and Cognition Laboratory, Department of Experimental Psychology, University of Oxford, South Parks Road, Oxford, OX1 3UD, UK. e-mail: kia.nobre@psy.ox.ac.uk

Received: 06 December 2007; paper pending published: 12 December 2007; accepted: 14 January 2008; published online: 28 March 2008.

Citation: Front. Hum. Neurosci. (2008) 1: 4. doi: 10.3389/neuro.09.004.2007

Copyright (c) 2008 Nobre, Griffin and Rao. This is an open-access article subject to an exclusive license agreement between the authors and the Frontiers Research Foundation, which permits unrestricted use, distribution, and reproduction in any medium, provided the original authors and source are credited.
Visual short-term memory (VSTM) provides a suitable entry point for the investigation of this basic and important issue. Most views of VSTM emphasize its limitations and stability rather than its flexible control. VSTM is highly limited in its capacity (Cowan, 2000; Davis and Holmes, 2005; Luck and Vogel, 1997; Wheeler and Treisman, 2002; Xu and Chun, 2006). Its contents, to a large extent, are already the product of focused attention (Atkinson and Shiffrin, 1971; Schmidt et al., 2002), and are considered to remain stable during the maintenance period across saccadic eye movements or distraction (Irwin, 1992; Irwin and Andrews, 1996; Phillips, 1974). Retrieval from VSTM is usually thought to proceed in an automatic and serial fashion (Sternberg, 1966, 1975).

However, a growing number of studies have recently begun to challenge this rigid view VSTM. Studies using retrodictive cues ("retro-cues") to orient attention to particular locations or objects within arrays held in VSTM have reported consistent performance benefits in change-detection and memory-search tasks (Griffin and Nobre, 2003; Landman et al., 2003; Lepsien and Nobre, 2007; Lepsien et al., 2005; Makovski and Jiang, 2007; Makovski et al., 2006, 2008; Matsukura et al., 2007; Nobre et al., 2004; Sligte et al., 2008). The mechanisms by which attentional orienting enhances VSTM functions are just beginning to be explored. Work so far has ruled out possible uninteresting, artifactual explanations for the effects, such as: speed-accuracy trade-offs (Griffin and Nobre, 2003; Lepsien et al., 2005), response biases (Griffin and Nobre, 2003), eye movements (Griffin and Nobre, 2003; Matsukura et al., 2007), or articulation

${ }^{1}$ Short-term memory (STM) is defined here simply as the set of processes by which stimulus-related representations, in the absence of external cues, are maintained, manipulated and accessed over brief retention intervals (seconds). The terminology was chosen for being neutral with respect to specific theoretical positions. STM is often used interchangeably with the term "working memory", but the former makes no specific assumption with regard to whether STM and long-term memory involve separate or independent memory stores (see Ranganath and Blumenfeld, 2005). 
(Makovski and Jiang, 2007; Makovski et al., 2006, 2008; Matsukura et al., 2007). Several, more interesting, possibilities remain, which are not mutually exclusive. To date, studies have investigated how attentional orienting may influence the maintenance of items in VSTM. Some studies have suggested that attentional orienting may enhance the active maintenance of relevant items and/or suppress maintenance of irrelevant items (Lepsien and Nobre, 2006, 2007). Other studies have suggested that attentional orienting may protect the maintenance of specific items from decay or proactive interference (Makovski and Jiang, 2007; Makovski et al., 2006; Matsukura et al., 2007; Sligte et al., 2008).

The present study complements and extends this growing literature, by exploring the ability of spatial attentional orienting to optimize VSTM retrieval functions. Specifically, we exploited the high temporal resolution and functional sensitivity of event-related potentials (ERPs) to test the ability of spatial orienting to optimize the retroactive search through VSTM for a target probe. Spatial orienting was manipulated by providing spatially informative (spatial) or non-informative (neutral) retro-cues presented 1500-2500 ms after arrays containing different VSTM loads (1, 2 or 4 colored items) and before the target probe (similar to Lepsien et al., 2005). As is well established, in the absence of attentional orienting, response times (RTs) for searching for items within VSTM increases linearly with load (Sternberg, 1966, 1975). Eventrelated potentials elicited by the probe stimulus in the neutral retro-cue trials also provided a direct neural correlate of retroactive search through VSTM. We discovered a new ERP component, which we termed $\mathrm{N} 3_{\mathrm{RS}}$, specifically linked to retroactive search through VSTM. Both the amplitude and duration of the $\mathrm{N} 3_{\mathrm{RS}}$ increased monotonically with VSTM load.

If spatial orienting is able to optimize the search path through VSTM and prioritize retrieval of the item at the relevant location, the decrement of RT over VSTM load should be attenuated. Furthermore, if spatial orienting provides an effective short-cut to the relevant item, its effects should become increasingly pronounced as VSTM load increases. Attenuation of the $\mathrm{N} 3_{\mathrm{RS}}$ ERP component by spatial retro-cues would provide the strongest direct evidence for modulation of search-related neural activity, ruling out alternative explanations for the behavioral effects. Finding that attentional orienting can change the search path through VSTM would pose a strong challenge to standard theories of VSTM.

\section{MATERIALS AND METHODS \\ Human subjects}

Twenty-four healthy right-handed (Oldfield, 1971) subjects (age range 18-23 years, 9 females) took part in the experiment as paid volunteers. Visual acuity was normal or corrected to normal. The final behavioral and ERP analyses included data from a subset of 16 participants who showed above-chance performance (4 participants excluded) and had a sufficient number of artifact-free ERP trials (4 additional participants excluded) across all task conditions. The experimental methods were non-invasive and had ethical approval from the Department of Experimental Psychology, University of Oxford, UK.

\section{Stimuli and task}

The task is illustrated in Figure 1. Participants viewed arrays of one, two or four differently colored tilted crosses ( $x$ 's) followed by a spatially informative or neutral retro-cue; and made a delayed decision about the color of the items in the array. The participants' task was to decide whether a probe stimulus, presented at the end of the trial, had been present in the array. There were two types of trial. In "spatial" trials, the retro-cue indicated the critical spatial location within the remembered array with $100 \%$ validity. In "neutral" trials, the retro-cue provided no spatial information. In both trial types the retro-cue was presented 1500-2500 ms after the array.

Each trial contained the same sequence of events. The trial began with a square (side length $0.8^{\circ}$ ) appearing at the centre of the screen for a period that ranged randomly between 600 and $900 \mathrm{~ms}$. An array of differently colored tilted crosses then appeared for $100 \mathrm{~ms}$. The colors of the crosses were randomly selected from: red, blue, green, yellow,

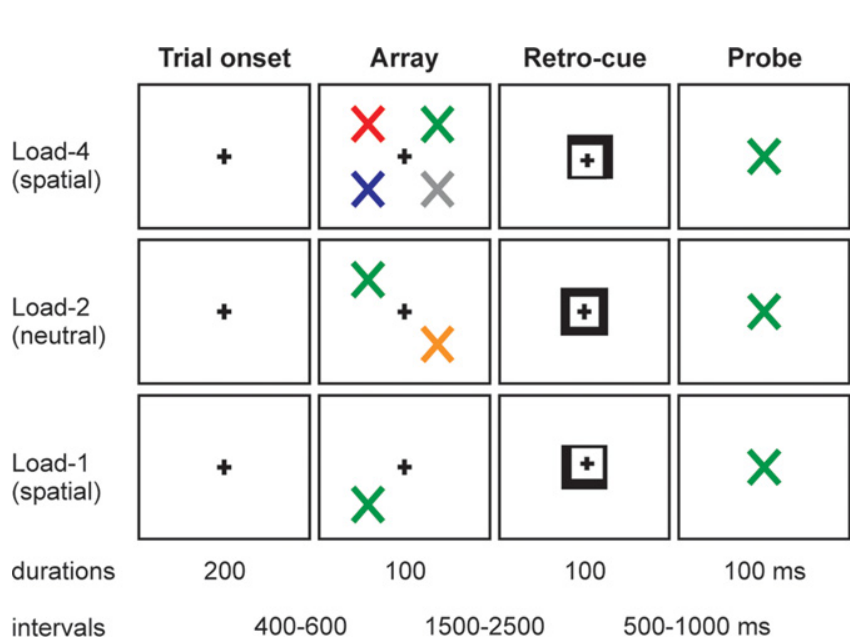

Figure 1. Schematic of the experimental task, with examples of trials of different loads $(1,2,4)$ and different types of retro-cue (neutral, spatial).

orange, cyan, pink, grey. Each cross was $0.8^{\circ}$ visual angle in size, and centered at $3^{\circ}$ horizontal and $3^{\circ}$ vertical eccentricity. The array could contain 1, 2 or 4 crosses (33\% probability of each number of crosses). If the array contained 2 crosses, they were always presented along a diagonal (top-left and bottom-right, or bottom-left and top-right). If the array contained 4 crosses, there was 1 cross in each quadrant. After a random interval varying between 1500 and $2500 \mathrm{~ms}$, a retrocue appeared for $100 \mathrm{~ms}$. In spatial trials (67\% of total trials) the cue consisted of two adjacent sides of the square brightening (forming an arrow), which instructed the subject to attend covertly to that location in the VSTM representation of the array (e.g., top-right). When only 1 or 2 items had been present in the array, the cue would always point to a location where an item had been (i.e., it never pointed to a previously empty position). In neutral trials ( $33 \%$ of total trials) the cue consisted of the whole square brightening, which imparted no spatial information. After a random interval between 500 and $1000 \mathrm{~ms}$, a colored-cross probe stimulus appeared in the centre of the screen for $100 \mathrm{~ms}$. The probe stimulus could appear in any of the eight possible colors, and the task was to decide whether the color of the probe stimulus had been present or absent from the array. When the probe stimulus had been present in the array, the spatial retro-cues retrodicted its correct location $100 \%$ of the time. Subjects responded by pressing the left button of the response box if the probe stimulus did appear in the array, and the right button if the probe stimulus did not appear in the array.

The probe stimulus was present or absent with equal probability $(50 \%)$. This was true for both spatial and neutral trials, and whether there had been 1, 2 or 4 items in the array. Within spatial and neutral trials, the three levels of load (1, 2, 4 items) occurred equally frequently. Within spatial trials, the four cue directions (top-left, top-right, bottom-left, bottom-right) occurred equally frequently. The number of trials in each experimental condition is broken down in Table $\mathrm{S} 1$ of the supplementary materials. There were sixteen blocks of trials in the experiment, plus one additional practice block at the beginning.

\section{Procedure}

Participants were comfortably seated in a dimly illuminated, electrically shielded room, facing a computer monitor placed $100 \mathrm{~cm}$ in front of them. They were informed, at the beginning of the experiment, about the relationship between the cue, array and probe stimuli. They were instructed to maintain fixation on a small fixation marker that was continuously present at the centre of the monitor, and to respond to the probe stimulus as quickly as possible, while avoiding mistakes. The hand of response was counterbalanced between subjects. Participants were 
also asked to avoid blinking and to suppress saccades throughout the experiment.

\section{Behavioral analyses}

The main goal of the behavioral analyses was to determine whether spatial retro-cues could facilitate retrieval from VSTM in face of increasing memory loads. The most relevant results were therefore those involving probe-present trials. In these trials, subjects can terminate the search and make decisions based on information retrieved from the location of the probe in the previous array. In the case of spatially cued trials, the cue effectively defines the relevant location. Though in principle spatia cueing can also restrict visual search to one location in probe-absent trials, previous work has shown that spatial retro-cueing is not as effective (Lepsien et al., 2005). Instead, subjects engage in a more exhaustive search in both spatially cued and neutral trials. All the behavioral data are presented in the supplementary materials, but analysis concentrated on probe-present trials.

Accuracy and median reaction time (RT) to respond to probe stimuli were analyzed by repeated-measures analyses of variance (ANOVAs). Both analyses tested the factors of Retro-cue (spatial, neutral) and memory Load (1, 2, 4 items). Only correct responses were included in the $\mathrm{RT}$ analysis. Of main interest were interactions between retro-cue and load. Post-hoc comparisons of means of medians were also carried out to guide interpretation where appropriate. To determine whether spatial retro-cues diminished the RT slope for retrieving a probe stimulus among increasing memory load, the interaction between retro-cue and the linear contrast of load was investigated. In addition, the RT/load slope for spatial and neutral probe-present trials was calculated by fitting a regression line through median RTs at the three loads. Slopes in the two conditions were compared using a paired-samples $t$-test. The Greenhouse-Geisser epsilon correction for non-sphericity was applied where appropriate (Jennings and Wood, 1976), and only corrected probability values and degrees of freedom are reported.

\section{ERP recording}

The EEG was recorded continuously using EEG-8 amplifiers (Contact Precision Instruments, London) from 60 scalp sites using non-polarizable tin electrodes mounted on an elastic cap (Electro-Cap Inc.), positioned according to the 10-20 International system (AEEGS, 1991). The montage included eight midline sites (FPZ, FZ, FCZ, CZ, CPZ, PZ, POZ, and OZ) and 26 sites over each hemisphere (FP1/FP2, AF3/AF4, AF7/AF8, F1/F2, F3/ F4, F5/F6, F7/F8, FC1/FC2, FC3/FC4, FC5/FC6, FT7/FT8, C1/C2, C3/C4, C5/C6, T7/T8, CP1/CP2, CP3/CP4, CP5/CP6, TP7/TP8, P1/P2, P3/P4, P5/ P6, P7/P8, P03/P04, P07/P08, and 01/02). Additional electrodes were used as ground and reference sites. The EEG was referenced to the right mastoid, then re-referenced off-line to the algebraic average of the right and left mastoids. The signal was amplified 20,000 times and digitized at a sampling rate of $250 \mathrm{~Hz}$. Data were recorded with a band-pass filter of $0.03-100 \mathrm{~Hz}$. The epoching of ERPs was performed off-line. Epochs started $200 \mathrm{~ms}$ before and ended $600 \mathrm{~ms}$ after stimulus onset. The prestimulus baseline of $200 \mathrm{~ms}$ was used for all analyses.

Horizontal and vertical eye movements were detected by recording the horizontal and vertical electrooculogram (HEOG and VEOG) bipolarly with electrodes placed around the eyes. Eye movements and gaze position were also monitored using an infrared video-based eye tracker with theoretical resolution around $0.1^{\circ}$ (iView, SMI). Eye movements or deviations from central fixation were detected with the eye tracker using an algorithm that calculated sites and duration of fixation points during each trial using infrared tracking. Trials with fixation points further than $1^{\circ}$ apart from central fixation were excluded from the analysis.

Epochs containing excessive noise or drift $( \pm 100 \mu \mathrm{V})$ at any electrode between -200 and $+600 \mathrm{~ms}$ were excluded. In addition, epochs with eye-movement artifacts (blinks or saccades) were rejected. Blinks were identified as large deflections $( \pm 50 \mu \mathrm{V})$ in the HEOG or VEOG electrodes. Finally, trials with incorrect behavioral responses were discarded. To maintain an acceptable signal-to-noise ratio, a lower limit of 15 artifactfree trials per subject per condition was set.

\section{ERP analysis}

The ERP analysis focused on data from probe-present (yes) trials, where VSTM search could be terminated upon the successful retrieval of the relevant item. Separate ERP averaged waveforms were constructed for probes in spatial $(\mathrm{S})$ and neutral $(\mathrm{N})$ trials at each memory load $(1 / 2 / 4)$, resulting in six ERP conditions: Spatial/Load-1 (S1), Spatial/Load-2 (S2), Spatial/Load-4 (S4), Neutral/Load-1 (N1), Neutral/Load-2 (N2), and Neutral/Load-4 (N4).

A topographical analysis using the Cartool software (Brunet, Functional Brain Mapping Laboratory, Geneva, Switzerland) was used to segment the grand-averaged ERP waveforms from the six experimental conditions into successive periods of stable electric field patterns, corresponding to dissociable functional states of the brain (Michel et al., 1992; Pascual-Marqui et al., 1995). For the segmentation analysis, ERPs for each subject and condition were recalculated against the average reference and normalized to their global field power (GFP) (Lehmann, 1987) before group averaging. The dominant scalp topographies appearing in the group averaged ERP from each condition over time were identified. This analysis was completed from 0-600 ms after stimulus onset, with the constraint that a given scalp topography lasted at least $10 \mathrm{~ms}$, and that maps should not be more than $98 \%$ correlated. The optimal number of maps that best explained the entire data set was defined by a crossvalidation criterion (Pascual-Marqui et al., 1995).

The mean amplitudes of the identifiable potentials within periods of stable topographical topographies were measured over the electrode locations and time periods where they were most prominent. Repeatedmeasures ANOVAs tested for the effects of spatial orienting by retro-cue (spatial, neutral) and load $(1,2,4)$ upon the identifiable ERP potentials. Additional factors related to electrode location along the anterior-toposterior (region) and left-to-right (side) axes were also included as necessary. The Greenhouse-Geisser epsilon correction for non-sphericity was applied where appropriate (Jennings and Wood, 1976).

In addition, an exploratory approach was used to identify the time course and spatial distribution of brain activity affected by memory load. Successive $t$-tests for each data point between 0 and $600 \mathrm{~ms}$ were calculated, comparing the load-4 condition to the load-2 condition in neutral trials (Doherty et al., 2005; Guthrie and Buchwald, 1991; Murray and Jones, 2002). Effects were considered significant if a $p<0.05$ two-tailed criterion was exceeded for $40 \mathrm{~ms}$ (10 data points) over at least three adjacent electrodes. No effects, over and above those occurring within identifiable components, emerged.

\section{RESULTS}

Data from the sixteen subjects with good behavioral performance and clean ERP data were used to characterize both the behavioral and ERP effects. Behavioural and ERP results converged to demonstrate that spatial orienting optimized search for a target item within VSTM.

\section{Behavioral effects}

As expected, increases in VSTM load in neutral retro-cue trials resulted in performance decrements in both accuracy and RT measures. In agreement with our predictions, spatial retro-cues significantly attenuated the load effects in both accuracy and RTs.

\section{Effects on performance accuracy}

Figure 2A plots the accuracy results for probe-present conditions. The full set of data, across probe-present and probe-absent trials is included in Tables S2 and S3 of the supplementary material. The mean percentage of correct responses in probe-present trials was $89 \%$. Both retro-cue and load exerted significant main effects on performance accuracy $\left(F_{(1,15)}=28.10\right.$, $p<0.001$ and $F_{(1.7,26.4)}=56.93, p<0.001$ respectively). Overall, subjects were more accurate in spatial retro-cue trials $(92 \%)$ compared to neutral 

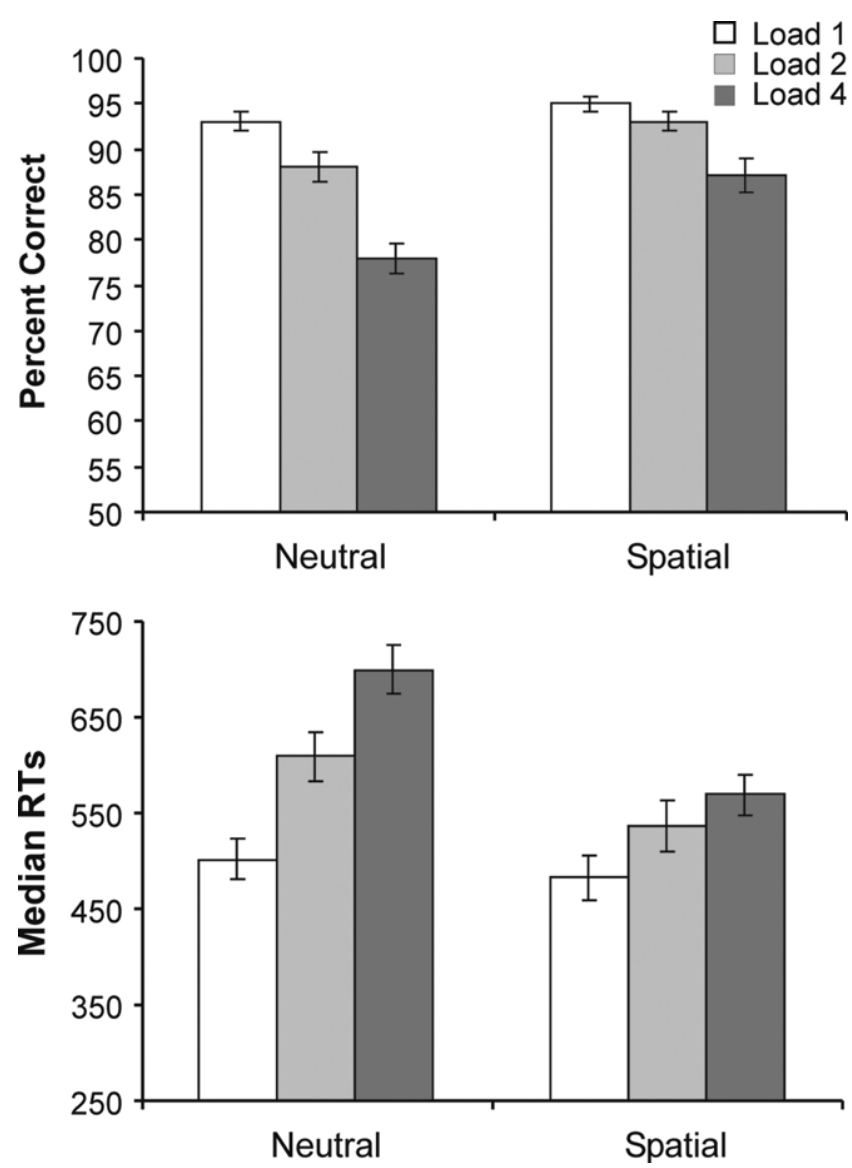

Figure 2. Percent correct (above) and averaged median RTs (below) in probe present trials across the two types of retro-cue (neutral, spatial) and three working-memory loads $(\mathbf{1}, \mathbf{2}, \mathbf{4})$. Error bars show standard errors of the means.

trials (86\%); and became increasingly inaccurate as load increased (load$1=94 \%$, load $-2=91 \%$, load $-4=82 \%$ ). The linear decrease in performance with increasing load was supported by a significant linear contrast of load $\left(F_{(1,15)}=28.10, p<0.001\right)$.

The modulation of the accuracy of VSTM search by spatial retro-cues was indicated by a significant interaction between the retro-cue and load factors $\left(F_{(1.8,26.3)}=5.22, p=0.02\right)$, which showed that the effect of load upon performance was greater in the neutral trials than in spatial trials. The differing linear effects of load in the two retro-cue conditions was confirmed by an interaction between retro-cue and the linear contrast of load $\left(F_{(1,15)}=80.54, p<0.001\right)$. Post-hoc one-tailed $t$-tests showed that performance was better in spatial trials compared to neutral trials at al three array loads [load-1: $t(15)=2.15, p=0.02$; load-2: $t(15)=2.81$, $p=0.005$; load-4: $t(15)=4.30, p<0.001]$. The effects of retro-cue on accuracy also became accentuated as load increased. Paired, one-tailed, $t$-tests showed that the difference between the percent correct responses for neutral vs. spatial retro-cues tended to be larger for load-2 ( $m=4.6 \%$, $\mathrm{SE}=1.6 \%)$ than load $-1(m=2.0 \%, \mathrm{SE}=0.9 \%)[t(15)=1.45, p=0.084]$, and was significantly larger for load-4 $(m=9.2 \%, \mathrm{SE}=2.1 \%)$ than load$2[t(15)=1.79, p=0.047]$.

\section{Effects on response times}

Effects on response times complemented those on accuracy, and ruled out any influence of speed-accuracy trade-off. Figure 2B plots the $\mathrm{RT}$ results for probe-present conditions. The speed of responses varied significantly according to retro-cue $\left(F_{(1,15)}=42.01, p<0.001\right)$ and load $\left(F_{(1.5,22.0)}=61.67, p<0.001\right)$. Responses were significantly faster in spatial trials $(530 \mathrm{~ms})$ compared to neutral trials $(604 \mathrm{~ms})$; and became progressively slower as the array load increased (load- $1=493 \mathrm{~ms}$, load $-2=573 \mathrm{~ms}$, load $-3=634 \mathrm{~ms}$ ). The linear increase in median RTs over load was confirmed by a significant linear contrast of load $\left(F_{(1,15)}=79.83, p<0.001\right)$.

As with accuracy, the decrements in RTs as a function of load in VSTM were significantly and increasingly attenuated by spatial retrocues. The effect of load on RT differed according to the retro-cueing condition, as revealed by a significant interaction between retro-cue and load $\left(F_{(1.6,24.7)}=18.23, p<0.001\right)$ and between retro-cue and the linear contrast of load $\left(F_{(1,15)}=25.47, p<0.001\right)$. Post-hoc one-tailed $t$-tests showed that the median RTs were significantly faster in spatial trials compared to neutral trials for arrays of load-2 $[t(15)=4.62, p<0.001]$ and load- $4[t(15)=6.80, p<0.001]$. At load -1 , the effect was marginal $[t(15)=1.68, p=0.06]$. The effects of retro-cue on RTs became accentuated as load increased. Paired, one-tailed, $t$-tests showed that the difference between RTs for neutral vs. spatial retro-cues was significantly larger for load-2 $(m=71.2 \mathrm{~ms}, \mathrm{SE}=15.4 \mathrm{~ms})$ than load- $1(m=18.8 \mathrm{~ms}$, $\mathrm{SE}=11.2 \mathrm{~ms})[t(15)=3.53, p=0.002]$, and for load $-4(m=131.1 \mathrm{~ms}$, $\mathrm{SE}=19.3 \mathrm{~ms})$ than load-2 $[t(15)=3.33, p=0.002]$.

To test directly whether spatial retro-cueing increased the efficiency of search through an array held within VSTM, the RT/load slopes were compared between the spatial and neutral conditions. Slopes were derived for each subject by computing the best fitting regression line over the three loads in spatial and neutral conditions. A paired-samples $t$-test showed that the RT/load slopes were significantly smaller in the spatial conditions ( $m=27 \mathrm{~ms} /$ item, $\mathrm{SE}=7.1 \mathrm{~ms}$ ) compared to the neutral conditions $(m=63 \mathrm{~ms} / \mathrm{item}, \mathrm{SE}=5.7 \mathrm{~ms})[t(15)=5.10, p<0.001]$.

\section{ERP effects}

Topographical and amplitude analyses of the ERPs identified a novel component, which reflected neural activity related to searching for a target retroactively in VSTM. The component had a frontal-central distribution, was negative in polarity, and started around $300 \mathrm{~ms}$, peaking around $320 \mathrm{~ms}$. We termed this component $\mathrm{N}_{\mathrm{RS}}$ for its polarity (Negative), approximate latency (300), and functional association (Retroactive Search).

ERP topographies. Figure 3 shows the results of the topographical segmentation of the ERPs. Probes in neutral retro-cue trials elicited a distinct topography starting around $300 \mathrm{~ms}$ and peaking around $320 \mathrm{~ms}$, whose duration increased proportionally with VSTM load ( $\mathrm{N}_{\mathrm{RS}}$, map-4). It was characterized by a large negative potential broadly distributed over frontal-central regions of the scalp and a positive potential over the midline posterior scalp. This topography was not observable in any of the spatial retro-cue conditions, suggesting a clear difference in the functional states triggered by probes in the neutral and spatial retro-cue conditions.

Prior to the $\mathrm{N}_{\mathrm{RS}}$, probes in both neutral and spatial retro-cue trials shared a common topography, which was characterized by a combination of the lateral posterior N1 visual potential and a positive midline P2 potential over frontal to posterior regions of the scalp (map 2). After the N1/P2 stage, neural processing of probes in the spatial retro-cue conditions was characterized only by a positive potential over the midline posterior scalp (P3e, map 3). This functional state (map 3) was also observed in the neutral retro-cue condition when only one item had been present in the array (N1), just preceding the $\mathrm{N} 3_{\mathrm{RS}}$ potential (map 4).

Following maps 3 and 4 , neural activity across all conditions was again characterized by the same two topographical patterns (maps 5 and 6), though the onset of these maps varied in a systematic way across conditions. Both of these maps ( 5 and 6 ) were characterized by a positive potential distributed over the midline posterior scalp. The maps were similar to that the immediately preceding topography in the spatial retro-cue conditions (map 3) (95\% correlation between maps 3 and 5 ) and to one another (97\% correlation between maps 5 and 6$)$. It is possible, therefore, that 


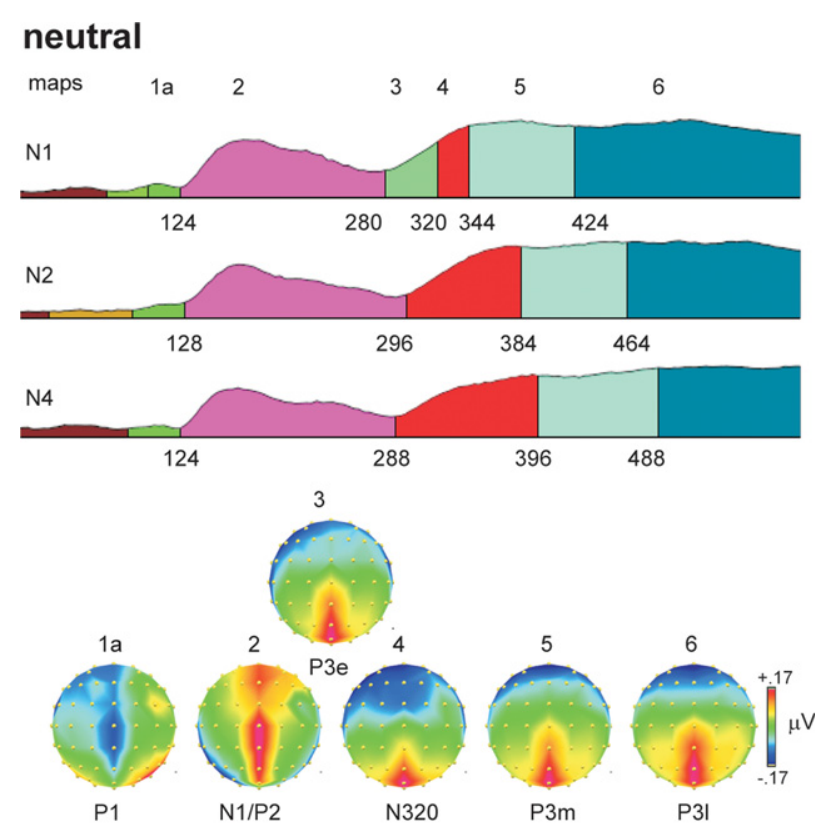

spatial
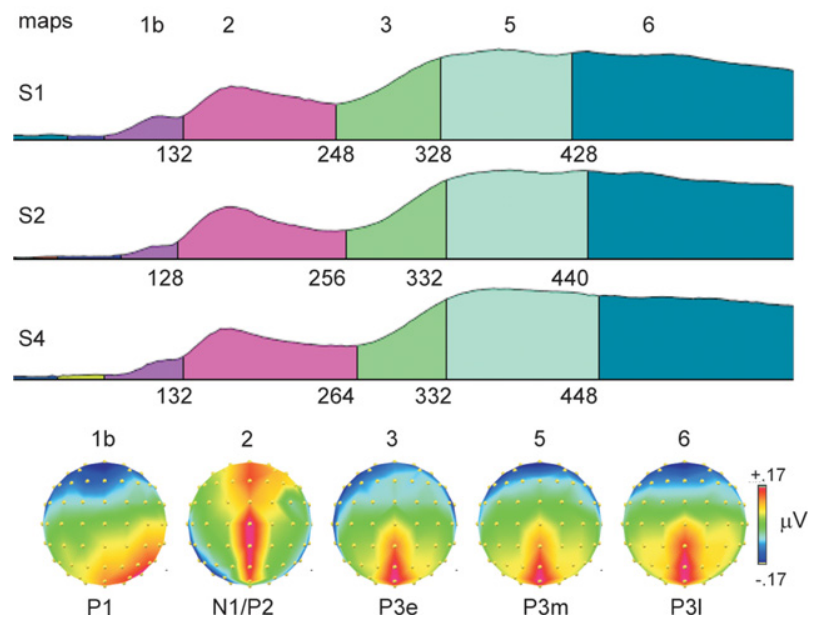

Figure 3. Topographical segmentation of grand-averaged waveforms in the six experimental conditions, according to type of retro-cue (neutral, spatial) and working-memory load $(\mathbf{1}, \mathbf{2}, 4)$. The global field power was used to normalize the waveforms, and is used as the template upon which the different topographical segments are displayed. Consistent topographical maps emerging after around $100 \mathrm{~ms}$ are displayed below the set of segmentations for the neutral retro-cue conditions (top panel) and spatial retro-cue conditions (bottom panel). Maps are color-coded, with equal colors representing equal topographies. Maps are also numbered above the colored segments in the GFP waveforms and above the corresponding topographical map. The onset latency for topographies (in ms) is provided below each GFP waveform. Topographies are plotted from a bird's-eye view, with the top corresponding to frontal electrode positions, and left corresponding to left positions. The normalized voltage scale (in microvolts), consistent across all maps, is shown to the right of the topographies, with maximum positive voltage as red and maximum negative voltage as dark blue. Because the topographies are derived from GFP-normalized waveforms, the amplitude range in the topographies is very narrow.

these maps represent subtle changes in neural activity, which has been chunked into three discrete phases. We have labeled these phases as P3early (P3e), P3-middle (P3m), and P3-late (P3I). Over time, the distribution of the midline posterior positivity can be observed to spread laterally, and the negativity over frontal sites to spread posteriorly. The onset latencies of the last two maps in the group data show the same pattern of variation as the RTs. Bivariate Pearson's correlation analyses using the onset latencies of these topographies and the average median RTs in the six conditions show strong significant correlations between these neural stages and response times (P3m and RT: $r(4)=0.87, p<0.05$; P3I and $\mathrm{RT}$ : $r(4)=0.99$, $p<0.001)$. Complementary negative correlations were found with accuracy measures (P3m and percent-correct: $r(4)=-0.78, p=0.07$; $\mathrm{P} 3 \mathrm{l}$ and percent correct: $r(4)=-0.93, p<0.01)$.

ERP amplitudes. Complementing the topographical segmentation, analysis of the amplitudes of ERP components showed a strong interaction between VSTM load and spatial orienting upon the mean amplitude of the $\mathrm{N}_{\mathrm{RS}}$. The grand-averaged ERP waveforms are shown in Figure 4. The $\mathrm{N3}_{\mathrm{RS}}$ was analyzed between 300 and $340 \mathrm{~ms}$, over midline and flanking electrodes (5 levels of electrode side) from frontal to parietal sites (five levels of region) - F3/1/Z/2/4, FC3/1/Z/2/4, C3/1/Z/2/4, $\mathrm{CP} 3 / 1 / \mathrm{Z} / 2 / 4, \mathrm{P} 3 / 1 / \mathrm{Z} / 2 / 4$. The analysis showed a clear interaction between retro-cue and VSTM load (simple interaction: $F_{(1.8 .27 .6)}=5.66, p<0.01$; interaction with linear contrast of load: $\left.F_{(1,15)}=11.92 . p<0.01\right)$. The effects were largest over the midline, and tended to be largest over more frontal regions (retro-cue $\times$ load $\times$ side: $F_{(3.8,56.9)}=3.72, p<0.01$; retrocue $\times$ load $\times$ region $\times$ side: $\left.F_{(4.3,64.9)}=2.34, p=0.06\right)$. Subsidiary analyses examining load effects for each of the retro-cue conditions revealed significant effects of load after neutral retro-cues (main effect of load $F_{(1.7,25.1)}=7.64, p<0.01$; linear contrast of load: $\left.F_{(1,15)}=20.09, p<0.001\right)$ but not after spatial retro-cues (main effect of load $F_{(1.9,27.9)}=0.46$, linear contrast of load $\left.F_{(1,15)}=0.60\right)$. The effects of load after neutral retro-cues were concentrated over midline electrodes and over the frontal-central scalp region (load $\times$ side: $F_{(3.6,54.4)}=3.94, p<0.01$; load $\times$ region $\times$ side: $\left.F_{(5.4,80.5)}=2.53, p<0.05\right)$.

Analysis of the $\mathrm{N} 3_{\mathrm{RS}}$ also revealed a strong effect of spatial orienting by retro-cues $\left(F_{(1,15)}=31.19, p<0.001\right)$. Waveforms after spatial retro-cues lacked the negative search-related potential, and were consequently significantly more positive. Instead, a broad positive potential with a parietal maximum had already started to develop in the spatial retro-cue condition, resulting in maximal differences over midline parietal electrode sites (retro-cue $\times$ region: $F_{(1.3,20.2)}=22.47, p<0.001$; retro-cue $\times$ side: $F_{(2.030 .6)}=19.94, p<0.001$; retro-cue $\times$ region $\times$ side: $\left.F_{(4.0 .60 .4)}=15.56, p<0.001\right)$.

Effects of VSTM load were not significant when independent of cueing, though there were statistical trends concentrated over midline frontal-central regions, according to the distribution of the $\mathrm{N} 3_{\mathrm{RS}}$ (main effect of load: $F_{(2.0,29.9)}=2.73, p=0.08$; linear contrast of load: $F_{(1,15)}=4.32$, $p=0.06$; load $\times$ side: $F_{(2.8 .41 .9)}=2.58, p=0.07$; load $\times$ region $\times$ side: $\left.F_{(5.4 .81 .2)}=2.30, p=0.05\right)$.

No significant interactions between Load and Retro-cues occurred before the $\mathrm{N}_{\mathrm{RS}}$. Analyses of the earlier ERP potentials are presented in the supplementary materials.

After the $\mathrm{N} 3_{\mathrm{RS}}$ time period, the topographies showed an equivalent distribution for all retro-cue and load conditions, characterized by a positive potential distributed around the parietal-occipital midline scalp. The late positive potential was analyzed between 360 and 400 ms over parietaloccipital and occipital regions, at midline and flanking electrodes - P03/Z/4 and $01 / Z / 2^{2}$.

Spatial orienting by retro-cues exerted the strongest effects during this period $\left(F_{(1,15)}=37.66, p<0.001\right)$. The potential was significantly more positive after spatial retro-cues, and the effect was focused over the parietal-occipital midline (retro-cue $\times$ region: $F_{(1,15)}=16.94, p<0.001$;

${ }^{2}$ Later activity, corresponding to the final and very similar topographic map, was not analyzed since activity in this period was likely to include factors that are not directly relevant to the current investigation - related to response execution (which occurred around the same time) and to control functions to be applied to successive trials based on current trial conflict and performance. 
neutral

N1 N2 N4
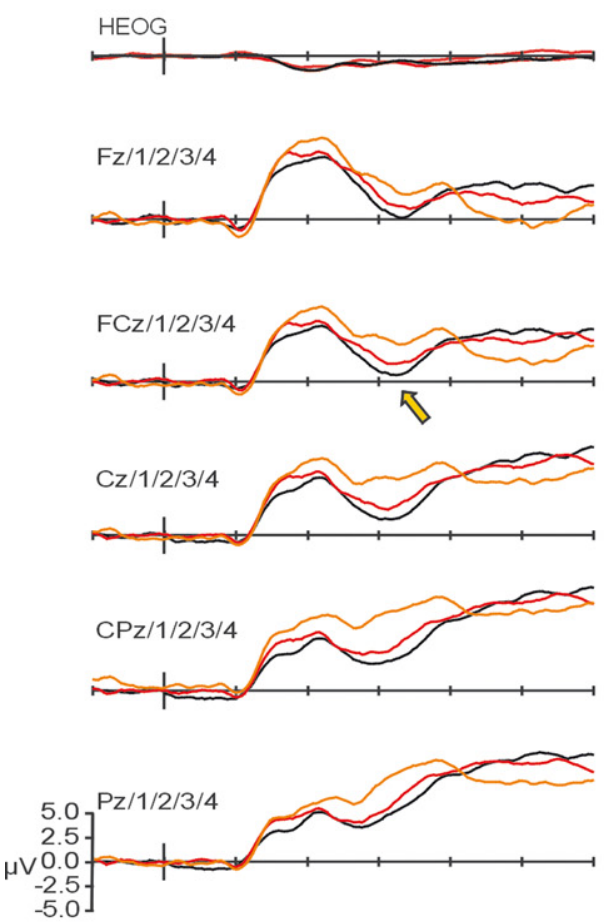

$\begin{array}{llllllll}-100 & 0.0 & 100 & 200 \quad 300 & 400 & 500 \quad 600 \mathrm{~ms}\end{array}$ spatial

S1 $\quad$ S2 S4
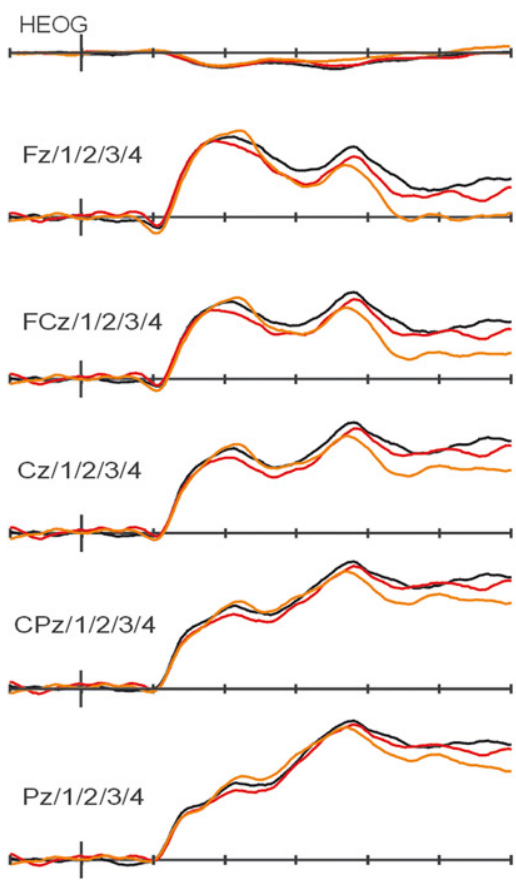

neutral $x$ spatial

S4 N4
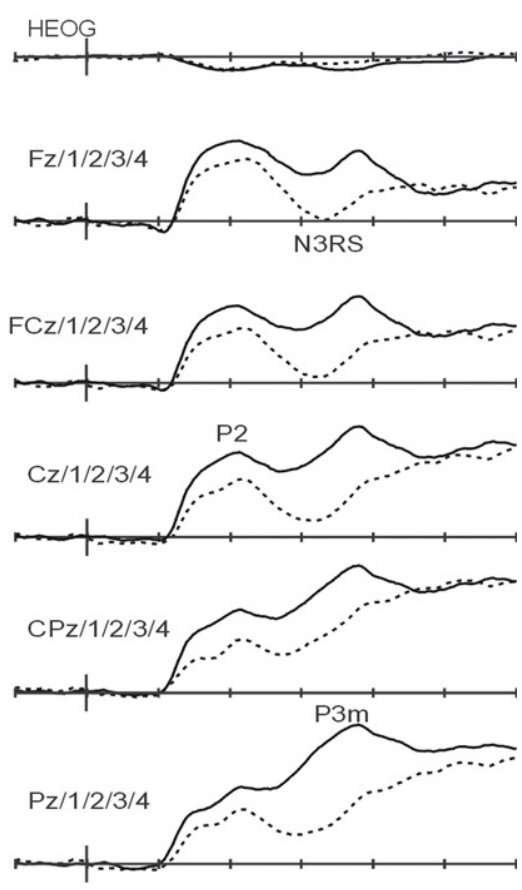

Figure 4. Grand-averaged waveforms, averaged over midline and flanking lateral electrodes used in statistical analyses. The left-most column shows the effects of working-memory load on ERPs elicited by probes after neutral retro-cues. Waveforms elicited after arrays of load-1 (N1, orange), load-2 (N2, red), and load-4 (N4, black) are superimposed. The effect of load on the $\mathrm{N}_{\mathrm{RS}}$ is indicated at FCz and flanking electrodes, by a yellow arrow. The middle column shows the effects of working-memory load on ERPs elicited by probes after spatial retro-cues. Waveforms elicited after arrays of load-1 (S1, orange), load-2 (S2, red), and load-4 (S4, black) are superimposed. The right-most column highlights the effects of retro-cue for load-4 conditions. Waveforms elicited by probes following neutral retro-cues (dashed) and spatial retro-dues (solid) are superimposed. Effects of spatial orienting by retro-cues occurred at P1, N1, $P 2, N 3_{R S}$, and $P 3$.

retro-cue $\times$ region $\times$ electrode: $\left.F_{(1.3,20.0)}=7.60, p=0.008\right)$. No effects of VSTM load approached significance, but there were remaining interactions between VSTM load and retro-cue, mainly over parietal-occipital electrodes (retro-cue $\times$ load $\times$ region: $F_{(2.0,29.7)}=4.56, p=0.02$; retrocue $\times$ linear contrast of load: $\left.F_{(1,15)}=3.79, p=0.07\right)$. Subsidiary analyses showed only focal effects of VSTM load after neutral retro-cues (load $\times$ region: $F_{(1.9,27.9)}=4.15 p=0.03$; main effect and linear contrast of load $F<1$ ). Effects of load after spatial retro-cues were far from significance $(F<1)$.

\section{DISCUSSION}

In this study, we used ERPs and behavioral measures to test the ability of spatial orienting to bias retrieval functions in VSTM. In particular, we manipulated VSTM load to identify neural activity specifically related to searching retroactively for an item within VSTM and to test its modulation by spatially retrodictive retro-cues. Our experimental approach was successful in identifying behavioral and ERP correlates of search-related activity, which varied systematically as a function of VSTM load. Our results yielded clear evidence that spatial orienting can influence search in VSTM directly.

The behavioral pattern of results was consistent with previous studies showing increases in accuracy and decreases in response time when predictive retro-cues are used to orient attention to relevant locations of arrays maintained in VSTM (Griffin and Nobre, 2003; Landman et al., 2003; Makovski and Jiang, 2007; Makovski et al., 2006, 2008;
Matsukura et al., 2007; Sligte et al., 2008). In particular, as in a previous study (Lepsien et al., 2005), spatial retro-cues attenuated the behavioral costs and diminished the RT slopes associated with retrieving probe items from progressively larger arrays (Sternberg, 1966), with more pronounced effects for probe-present compared to probe-absent trials.

Further analysis of the behavioral results showed that the effects of retro-cues became progressively accentuated as the load increased. Increasing effects of retro-cues in the context of increasing competition between items in VSTM is consistent with top-down attentional biasing of selective aspects of the maintained VSTM representation, such that the information related to the relevant item is enhanced and/or competition from irrelevant items is diminished. The pattern of results is consistent with that obtained in the previous fMRI study by Lepsien using a very similar task (Lepsien et al., 2005), but retro-cueing effects have not been found to increase significantly with load in other studies (Makovski et al., 2008; Matsukura et al., 2007). Makovski and colleagues (2008) found that the protective effects of retro-cues against proactive interference did not increase in magnitude beyond the case of load-1, in which attention is summoned automatically to the occupied location (though they do not analyze effects on response times, only on accuracy and sensitivity). They used their findings to argue against attentional biasing effects of retro-cueing, and to support mechanisms that protect selective aspects of the VSTM representation against interference. In the study by Matsukura and colleagues (2007), the number of items cued by the hemifield retro-cues increased as the VSTM load increased, making it difficult to draw inferences from the null effect of load. In the current 
experiment, the arrays were not subjected to proactive interference, and therefore it was not possible to test whether the increasing advantages for retro-cues in the context of larger VSTM loads would survive such a manipulation. Additional experiments will be required to determine the boundary conditions for VSTM load to influence the effects of retro-cues. Based on the current results, it is possible to conclude that retro-cues can trigger attentional biasing effects, at least in the absence of interfering stimulation. The conclusions are consistent with the observation that maintenance-related neural activity in specialized posterior brain regions is modulated after retro-cues (Lepsien and Nobre, 2006).

Analysis of ERPs triggered by the probe stimuli in the present experiment further showed that orienting attention to locations within VSTM arrays has direct consequences for the retrieval functions. By focusing our analysis on the topographies and amplitudes of the potentials triggered by probes present in VSTM arrays of different loads, we were able to identify a stage of neural processing associated with VSTM search and to chart its modulation by spatial orienting. Starting around $300 \mathrm{~ms}$, a fronto-centrally distributed negative potential was systematically related to the degree of retroactive search required to locate the probe within VSTM array $\left(\mathrm{N}_{\mathrm{RS}}\right)$. The topographical segmentation showed the duration of this functional state to increase as VSTM load increased in neutral retro-cue trials. In contrast, this functional state was completely absent when probes followed spatial retro-cues. The amplitude analysis showed the $\mathrm{N} 3_{\mathrm{RS}}$ to be increasingly negative as load increased after neutral retrocues, but found no significant modulation during this time period after spatial retro-cues.

The topographical differences during this period of neural processing suggest a difference in the kind of neural processing engaged by probes after neutral vs. spatial retro-cues. Topographical differences indicate activation of different neural generators or, at least, the differential weighting of activation in a common set of neural structures. The ERP findings are therefore compatible with spatial retro-cues enabling the direct retrieval of the item at the retrodicted location, and circumvention of VSTM search altogether. However, they are also compatible with a significant attenuation of search-related activity by spatial retro-cues, so that the brain areas participating in VSTM search do not contribute much to the scalp topography relative to other areas engaged in other, temporally overlapping functions.

The small, but still significant, residual behavioral effects of VSTM load in spatial retro-cue conditions attest to some degree of load-varying search even when spatial retro-cues specified the relevant location with $100 \%$ certainty. In order to settle whether spatial orienting modulates VSTM retrieval by circumventing vs. diminishing the amount of search, more behavioral testing will be informative. Using larger numbers of spatial retro-cue trials, it may be possible to characterize the distribution of response times. Bimodal distributions would suggest that participants may fail to use the spatial retro-cues on some trials. If so, it will be interesting to test how spatial retro-cues influence responses around each mode of the distribution to determine whether it is possible to eliminate load costs completely when retro-cues are used effectively.

As far as we can tell, the $\mathrm{N}_{\mathrm{RS}}$ is one of the first descriptions of an ERP marker for search through VSTM representations (see also Gomarus et al., 2006). Further experiments capitalizing on this kind of approach should prove fruitful in investigating the neural mechanisms and dynamics of VSTM retrieval more generally, as well as of its modulation by attentional orienting. At this stage, the modulation of the $\mathrm{N} 3_{\mathrm{RS}}$ by retro-cues clearly shows that the search path through VSTM is flexible and under voluntary control. The findings are compatible with two types of mechanisms. (1) Retro-cues could exert effects during the maintenance period, so that the nature of the maintained representation is changed to emphasize the relevant item and/or exclude the uncued items. As a consequence, the load of the maintained array in effect would shrink around the retro-cued item. According to this interpretation, when the probe item appears, it triggers a search through a VSTM array of reduced load. (2) Alternatively, or additionally, retro-cues could tag the relevant aspects of the VSTM representation for prioritized retrieval. When the probe is presented, it triggers a shift of attention to the relevant location or object, and in the case of a successful match, any additional search can be avoided.

Our findings suggest a direct action upon VSTM search and retrieval, in addition to any possible mechanisms during maintenance. The topographical segmentation revealed search-related activity only in the case of neutral retro-cues ( $\mathrm{N}_{\mathrm{RS}}$, map 4). When it was possible to orient attention to a relevant location, both in the case of spatial retro-cues and when only one item had been present in a neutral retro-cue trial, a different potential occurred, starting around $250 \mathrm{~ms}$, with a posterior parietal distribution (P3e, map 3). We speculate that this positive parietal potential may index spatial orienting of attention within a VSTM array and/or engaging with a selected target, perhaps in a fashion analogous to the N2PC, related to orienting attention to and/or selecting a relevant item from within a perceptual search array (Eimer, 1996; Hickey et al., 2006; Luck and Hillyard, 1994; Luck et al., 1997; Wascher and Wauschkuhn, 1996; Woodman and Luck, 2003). Unfortunately, it was not possible to separate trials into those where the relevant target item had occurred within the left vs. right visual field, in order to test whether this parietal potential varies in a way that is specific to the direction of spatial shifts. Future experiments will test directly the functional relation of this potential to spatial orienting within VSTM search, as well as its laterality.

Our results do not rule out the possibility that retro-cues also exerted top-down biasing effects during the period of VSTM maintenance. Indeed, we are partial to such possibility, but the current design was not optimally suited to test VSTM maintenance, since there was no direct ERP marker for maintenance that was independent from the physical appearance of the preceding arrays. Recently, an ERP marker of spatially lateralized maintenance-related delay activity has been described. After detection and encoding of a set of items to be maintained in VSTM, a negative posterior sustained potential develops over the contralateral scalp, termed CDA (contralateral delay activity) or SPCN (sustained posterior contralateral negativity) (Dell'Acqua et al., 2006; Klaver et al., 1999; Vogel and Machizawa, 2004). In perceptual search tasks, the CDA follows the N2PC, from which it can be dissociated on the basis of its latency and more medial topography (McCollough et al., 2007). Its magnitude varies systematically with VSTM load, becoming saturated as the load reaches an individual's capacity (Vogel et al., 2005). In order to test for direct effects of retro-cues on VSTM maintenance, it will be possible to measure modulation of the CDA triggered by lateralized arrays across several VSTM loads after spatial vs. neutral retro-cues. After neutral retro-cues, the CDA should continue to vary according to load. However, if retro-cues effectively diminish the maintained load, the differences in the CDA across load should be significantly reduced or eliminated.

Whereas the topographical segmentation in the present study was highly effective at pinpointing differences between functional neural states between conditions, it cannot point to the neural sources of these differences. The clear fronto-central distribution of the $\mathrm{N}_{\mathrm{RS}}$ would be consistent with the participation of prefrontal cortical areas, but other areas are also likely to form part of a network for searching through VSTM. The exact brain areas and their pattern of interaction may furthermore vary depending on the type of VSTM arrays implicated. Convergent data from methods with complementary high spatial resolution, such as fMRI and TMS, will prove especially useful to delineate the areas involved in VSTM search mechanisms. Many studies have examined the neural system involved in visual VSTM maintenance and retrieval generally, and have shown it to include prefrontal cortical areas, as well as posterior parietal and specialized occipital-temporal areas (Song and Jiang, 2005; Todd and Marois, 2004). However, less is known about areas specifically involved in searching through the contents of VSTM.

After search-related activity, in the case of neutral retro-cue trials, or the state we suggest is associated to attentional orienting in the case of spatial retro-cue trials, the waveforms display equivalent functional stages, which continue to be characterized by broadly distributed positive 
potentials that are maximal over midline parietal electrodes. The strong correlation between the onsets of these functional stages and reaction times, suggests that these potentials are closely linked to decision making and response selection that follow the retrieval of an item from the VSTM array.

\section{CONCLUSIONS}

Building on recent work suggesting a high degree of voluntary attentional control over VSTM, we have investigated the ability of spatial orienting to bias search for a target item within a VSTM representation. By varying the load of the VSTM arrays through which retroactive search was required, we were able to identify search-related behavioral measures and neural activity that varied systematically with load. In the absence of spatial orienting, behavioral accuracies and response times became increasingly impoverished as VSTM load increased. A new ERP marker of retroactive search through VSTM was also identified ( $\mathrm{N}_{\mathrm{RS}}$ ), whose duration and amplitude increased monotonically with the degree of search under increasing VSTM loads. Spatial orienting had large and significant effects upon both behavioral and ERP markers of load-varying VSTM search. Effects of load were attenuated or abolished by spatial retro-cues that retrodicted the relevant location for retrieving the target item within VSTM. The combined behavioral and ERP evidence showing that attentional orienting can optimize search through VSTM poses a strong challenge to standard theories emphasizing the stability and rigidity of VSTM.

\section{CONFLICT OF INTEREST STATEMENT}

The research was conducted in the absence of any commercial or financial relationships that could be construed as a potential conflict of interest.

\section{ACKNOWLEDGEMENT}

The research was supported by an award from the James S. McDonnell Foundation to ACN.

\section{SUPPLEMENTARY MATERIAL}

Distribution and number of trials across experimental conditions Both informative and neutral trials occurred interspersed in a random order throughout the experiment. There were 576 trials in total (384 spatial, 192 neutral; 192 with 1 item in the array, 192 with 2 items in the array, and 192 with four items in the array). Of the spatial trials, 192 were valid (stimulus was in the array, at the cued location), and 192 presented a probe stimulus that had not been present anywhere in the array. Of the neutral trials, 96 contained a probe that was in the array and 96 contained a probe that was not in the array. The minimum number of trials in any condition was 32 (32 trials for neutral cues at each memory load for each response condition) (see Table $\mathbf{S 1}$ ).

\section{Accuracy and RT results across all experimental conditions}

See Tables S2 and S3.

\section{REFERENCES}

AEEGS (1991). American electroencephalographic society guidelines for standard electrode position nomenclature. J. Clin. Neurophysiol. 8, 200-202.

Atkinson, R. C., and Shiffrin, R. M. (1971). The control of short-term memory. Sci. Am. 225, 82-90.

Averbach, E., and Coriell, A. S. (1961). Short-term memory in vision. Bell Syst. Tech. J. 40, 309-328.

Chambers, C. D., and Mattingley, J. B. (2005). Neurodisruption of selective attention: insights and implications. Trends Cogn. Sci. 9, 542-550 Epub:7 0ct 2005 (Review).

Cowan, N. (2000). The magical number 4 in short term memory: a reconsideration of mental storage capacity. Behav. Brain Sci. 24, 87-185.

Davis, G., and Holmes, A. (2005). The capacity of visual short-term memory is not a fixed number of objects. Mem. Cogn. 33, 185-195.

Dell'Acqua, R., Sessa, P., Jolicoeur, P., and Robitaille, N. (2006). Spatial attention freezes during the attention blink. Psychophysiology 43, 394-400.

Doherty, J. R., Rao, A., Mesulam, M. M., and Nobre, A. C. (2005). Synergistic effect of combined temporal and spatial expectations on visual attention. J. Neurosci. 25, 8259-8266.

Eimer, M. (1996). The N2pc component as an indicator of attentional selectivity. Electroencephalogr. Clin. Neurophysiol. 99, 225-234.

Gomarus, H. K., Althaus, M., and Wijers, A. A. (2006). The effects of memory load and stimulus relevance on the EEG during a visual selective memory search task: an ERP and ERD/ERS study. Clin. Neurophysiol. 117, 871-884.

Table S1. Number of trials per experimental condition according to the type of retro-cue (spatial, neutral), VSTM load (L-1, L-2, L-4), and correct response (probe-present, yes; probe-absent, no).

\begin{tabular}{lllllll}
\hline & L-1 (Yes) & L-2 (Yes) & L-4 (Yes) & L-1 (No) & L-2 (No) & L-4 (No) \\
\hline Spatial & 64 & 64 & 64 & 64 & 64 & 64 \\
Neutral & 32 & 32 & 32 & 32 & 32 & 32 \\
\hline
\end{tabular}

Table S2. Mean percent correct (and standard error) according to the type of retro-cue (spatial, neutral), working-memory load (L-1, L-2, L-4), and correct response (probe-present, yes; probe-absent, no).

\begin{tabular}{lllllll}
\hline & L-1 (Yes) & L-2 (Yes) & L-4 (Yes) & L-1 (No) & L-2 (No) & L-4 (No) \\
\hline Spatial & $95.1(0.87)$ & $93.1(1.06)$ & $86.9(1.87)$ & $94.8(1.16)$ & $92.9(1.50)$ & $84.0(2.26)$ \\
Neutral & $93.2(1.08)$ & $88.5(1.71)$ & $77.7(1.69)$ & $92.8(1.48)$ & $93.6(1.12)$ & $83.2(2.30)$
\end{tabular}

Table S3. Mean of the median reaction times (and standard error) according to the type of retro-cue (spatial, neutral), working-memory load (L-1, L-2, L-4), and correct response (probe-present, yes; probe-absent, no).

\begin{tabular}{lllllll}
\hline & L-1 (Yes) & L-2 (Yes) & L-4 (Yes) & L-1 (No) & L-2 (No) & L-4 (No) \\
\hline Spatial & $483.4(23.04)$ & $537.3(26.04)$ & $568.8(22.48)$ & $540.8(20.39)$ & $580.1(19.45)$ & $647.6(22.26)$ \\
Neutral & $501.3(20.96)$ & $608.5(25.22)$ & $700.0(25.46)$ & $579.8(19.66)$ & $617.2(15.86)$ & $676.4(20.88)$
\end{tabular}


Griffin, I. C., and Nobre, A. C. (2003). Orienting attention to locations in internal representations. J. Cogn. Neurosci. 15, 1176-1194.

Guthrie, D., and Buchwald, J. S. (1991). Significance testing of difference potentials Psychophysiology 28, 240-244.

Hickey, C., McDonald, J. J., and Theeuwes, J. (2006). Electrophysiological evidence of the capture of visual attention. J. Cogn. Neurosci. 18, 604-613.

Irwin, D. E. (1992). Memory for position and identity across eye movements. J. Exp. Psychol. Learn. Mem. Cogn. 18, 307-317.

Irwin, D. E., and Andrews, R. V. (1996). Integration and accumulation of information across saccadic eye movements. In Attention and Performance. XVI: Information Integration in Perception and Communication, T. Inui and J. L. McClelland eds (Cambridge, MA, MIT Press), pp. 125-155.

Jennings, J. R., and Wood, C. C. (1976). Letter: the epsilon-adjustment procedure for repeated-measures analyses of variance. Psychophysiology 13, 277-278.

Kastner, S., and Pinsk, M. A. (2004). Visual attention as a multilevel selection process. Cogn. Affect. Behav. Neurosci. 4, 483-500 (Review).

Klaver, P., Talsma, D., Wijers, A. A., Heinze, H. J., and Mulder, G. (1999). An event-related brain potential correlate of visual short-term memory. Neuroreport 13, 2001-2005

Landman, R., Spekreijse, H., and Lamme, V. A. (2003). Large capacity storage of integrated objects before change blindness. Vis. Res. 43, 149-164.

Lehmann, D. (1987). Principles of spatial analysis. In Handbook of electroencephalography and clinical neurophysiology. Vol. 1: Methods of analysis of brain electrical and magnetic signals, A. S. Gevins, A . Remond eds (Amsterdam, Elsevier), pp. 309-354.

Lepsien, J., Griffin, I. C., Devlin, J. T., and Nobre, A. C. (2005). Directing spatial attention in mental representations: interactions between attentional orienting and workingmemory load. Neuroimage 26, 733-743.

Lepsien, J., and Nobre, A. C. (2006). Cognitive control of attention in the human brain: insights from orienting attention to mental representations. Brain Res. 1105, 20-31.

Lepsien, J., and Nobre, A. C. (2007). Attentional modulation of object representations in working memory. Cereb. Cortex 17, 2072-2083.

Luck, S. J., Chelazzi, L., Hillyard, S. A., and Desimone, R. (1997). Neural mechanisms of spatial selective attention in areas V1, V2, and V4 of macaque visual cortex. J. Neurophysiol. 77, 24-42.

Luck, S. J., and Hillyard, S. A. (1994). Spatial filtering during visual search: evidence from human electrophysiology. J. Exp. Psychol. Hum. Percept. Perform. 20, 1000-1014.

Luck, S. J., and Hillyard, S. A. (2000). The operation of selective attention at multiple stages of processing: Evidence from human and monkey electrophysiology. In The New Cognitive Neurosciences, M. S. Gazzaniga ed. (Cambridge, MA, MIT Press), pp. 687-700.

Luck, S. J., and Vogel, E. K. (1997). The capacity of visual working memory for features and conjunctions. Nature 390, 279-281.

Luck, S. J., Woodman, G. F., and Vogel, E. K. (2000). Event-related potential studies of attention. Trends Cogn. Sci. 4, 432-441.

Makovski, T., and Jiang, Y. V. (2007). Distributing versus focusing attention in visual short-term memory. Psychonomic Bulletin \& Review, 14, 1072-1078.

Makovski, T., Shim, W. M., and Jiang, Y. V. (2006). Interference from filled delays on visual change detection. J. Vis. 6, 1459-1470.

Makovski, T., Sunsman, R., and Jiang, Y. V. (2008). Orienting attention in visual working memory reduces interference from memory probes. J. Exp. Psychol.: Learning memory, \& cognition, 34, 369-380.

Matsukura, M., Luck, S. J., Vecera, S. P. (2007). Attention effects during visual shortterm memory maintenance: Protection or prioritization? Percept Psychophys. 69, 1422-1434.

McCollough, A. W., Machizawa, M. G., and Vogel, E. K. (2007). Electrophysiological measures of maintaining representations in visual working memory. Cortex 43, 77-94.

Mesulam, M. M. (1999). Spatial attention and neglect: parietal, frontal and cingulate contributions to the mental representation and attentional targeting of salient extrapersonal events. Philos. Trans. R. Soc. Lond., B, Biol. Sci. 354, 1325-1346.

Michel, C. M., Henggeler, B., and Lehmann, D. (1992). 42-Channel potential map series to visual contrast and stereo stimuli: perceptual and cognitive event-related segments. Int. J. Psychophysiol. 12, 133-145.
Moore, T. (2006). The neurobiology of visual attention: finding sources. Curr. Opin. Neurobiol. 16, 159-165 Epub:24 Mar 2006 (Review).

Murray, A., and Jones, D. M. (2002). Articulatory complexity at item boundaries in serial recall: the case of Welsh and English digit span. J. Exp. Psychol. 28, 594-598.

Nobre, A. C. (2004). Probing the flexibility of attentional orienting in the human brain. In Cognitive Neuroscience of Attention, M. I. Posner, ed. (New York, NY, Guilford Press), pp. 157-179.

Nobre, A. C., Coull, J. T., Maquet, P., Frith, C. D., Vandenberghe, R., and Mesulam, M. M. (2004). Orienting attention to locations in perceptual versus mental representations. J. Cogn. Neurosci. 16, 363-373.

Oldfield, R. C. (1971). The assessment and analysis of handedness: the Edinburgh inventory. Neuropsychologia 9, 97-113.

Pascual-Marqui, R. D., Michel, C. M., and Lehmann, D. (1995). Segmentation of brain electrical activity into microstates: model estimation and validation. IEEE Trans Biomed. Eng. 42, 658-665.

Phillips, W. A. (1974). On the distinction between sensory storage and short-term visual memory. Percept. Psychophys. 16, 283-290.

Ranganath, C., and Blumenfeld, R. S. (2005). Doubts about double dissociations between short- and long-term memory. Trends Cogn. Sci. 9, 374-380 (Review).

Reynolds, J. H., and Chelazzi, L. (2004). Attentional modulation of visual processing. Annu. Rev. Neurosci. 27, 611-647 (Review).

Rushworth, M. F., and Taylor, P. C. (2006). TMS in the parietal cortex: updating representations for attention and action. Neuropsychologia 44, 2700-2716 Epub:7 Feb 2006 (Review)

Schmidt, B. K., Vogel, E. K., Woodman, G. F., and Luck, S. J. (2002). Voluntary and automatic attentional control of visual working memory. Percept. Psychophys. 64 754-763.

Serences, J. T., and Yantis, S. (2006). Selective visual attention and perceptual coherence. Trends Cogn. Sci. 10, 38-45 Epub:28 Nov 2005 (Review).

Sligte, I. G., Scholte, H. S., and Lamme, V. A. (2008). Are there multiple visual short-term memory stores? PLOS ONE. 3, e1699.

Song, J. H., and Jiang, Y. (2005). Connecting the past with the present: how do humans match an incoming visual display with visual memory? J. Vis. 5, 322-330.

Sperling, G. (1960). Negative afterimage without prior positive image. Science 131, 1613-1614.

Sternberg, S. (1966). High-speed scanning in human memory. Science 153, 652-654.

Sternberg, S. (1975). Memory scanning: new findings and current controversies. Q. J. Exp. Psychol. 27, 1-32.

Todd, J. J., and Marois, R. (2004). Capacity limit of visual short-term memory in human posterior parietal cortex. Nature 428, 751-754.

Vogel, E. K., and Machizawa, M. G. (2004). Neural activity predicts individual differences in visual working memory capacity. Nature 428, 748-751.

Vogel, E. K., McCollough, A. W., and Machizawa, M. G. (2005). Neural measures reveal individual differences in controlling access to working memory. Nature 438, 500-503.

Walter, W. G., Cooper, R., Aldridge,V. J., McCallum, W. C., and Winter,A. L. (1964). Contingent negative variation: an electric sign of sensorimotor association and expectancy in the human brain. Nature 203, 380-384.

Wascher, E., and Wauschkuhn, B. (1996). The interaction of stimulus- and response-related processes measured by event-related lateralizations of the EEG. Electroencephalogr. Clin. Neurophysiol. 99, 149-162.

Wheeler, M. E., and Treisman, A. M. (2002). Binding in short-term visual memory. J. Exp. Psychol. Gen. 131, 48-64.

Womelsdorf, T., and Fries, P. (2006). Neuronal coherence during selective attentional processing and sensory-motor integration. J. Physiol. 100, 182-193 Epub:17 Jan 2007 (Review).

Woodman, G. F., and Luck, S. J. (2003). Dissociations among attention, perception, and awareness during object-substitution masking. Psychol. Sci. 14, 605-611.

$\mathrm{Xu}, \mathrm{Y}$., and Chun, M. M. (2006). Dissociable neural mechanisms supporting visual shortterm memory for objects. Nature 440, 91-95 Epub:28 Dec 2005. 